\title{
Direct regulation of caspase-3 by the transcription factor AP-2 $\alpha$ is involved in aspirin-induced apoptosis in MDA-MB-453 breast cancer cells
}

\author{
FENG YAN ${ }^{1 *}$, QIONGZHI HE ${ }^{1 *}$, XIANG HU $^{1 *}$, WEN LI $^{1}, \mathrm{KE} \mathrm{WEI}^{1}, \mathrm{LI} \mathrm{LI}^{1}$, \\ YINGLI ZHONG $^{2}$, XIAOFENG DING ${ }^{1}$, SHUANGLIN XIANG ${ }^{1}$ And JIAN ZHANG ${ }^{1,3}$
}

\begin{abstract}
${ }^{1}$ Key Laboratory of Protein Chemistry and Development Biology of State Education Ministry of China, College of Life Sciences, Hunan Normal University, Changsha, Hunan $410081 ;{ }^{2}$ College of Bioscience and Biotechnology, Hunan Agricultural University, Changsha, Hunan 410128; ${ }^{3}$ Model Organism Division, E-Institutes of Shanghai Universities, Shanghai Jiao Tong University, Shanghai 200240, P.R. China
\end{abstract}

Received September 29, 2012; Accepted December 11, 2012

DOI: $10.3892 / \mathrm{mmr} .2013 .1257$

\begin{abstract}
Aspirin has been reported to trigger apoptosis in various cancer cell lines. However, the detailed mechanisms involved remain elusive. The present study aimed to investigate whether aspirin plays a role in apoptosis of MDA-MB-453 cells. The effect of aspirin on the proliferation of human MDA-MB-453 cells breast cancer cells was evaluated using MTT assay, flow cytometry and western blotting. The present study reports that aspirin induces the apoptosis of MDA-MB-453 breast cancer cells which was attributed to the increased expression and activation of caspase- 3 . Moreover, AP- $2 \alpha$, a transcription factor highly expressed in MDA-MB-453 cells, was identified as a negative regulator of caspase- 3 transcription and AP- $2 \alpha$ was attenuated following aspirin treatment. Therefore, aspirin may increase the expression of caspase- 3 by inducing the degradation of AP- $2 \alpha$, which increases activated caspase- 3 expression, thereby triggering apoptosis in MDA-MB-453 cells. Thus, aspirin may be used in breast cancer therapy.
\end{abstract}

\section{Introduction}

Aspirin (acetylsalicylic acid) has been used as a treatment for high body temperatures, headaches and muscle pain for more

Correspondence to: Dr Shuanglin Xiang or Dr Jian Zhang, Key Laboratory of Protein Chemistry and Development Biology of State Education Ministry of China, College of Life Sciences, Hunan Normal University, No.36 Lushan Road, Changsha, Hunan 410081, P.R. China

E-mail: xshlin@hunnu.edu.cn

E-mail: zhangjian@hunnu.edu.cn

*Contributed equally

Key words: aspirin, AP-2 $\alpha$, caspase-3, apoptosis, MDA-MB-453 than a century. Accumulating evidence from large-scale epidemiological studies, animal models and in vitro experiments suggest that regular use of aspirin may reduce the risk of cancer by inducing cancer cell apoptosis (1-3). Findings of recent studies have indicated that aspirin is able to block $\mathrm{NF}-\kappa \mathrm{B}$ activation $(4,5)$, induce iNOS to produce $\mathrm{NO}(6,7)$, inhibit COX-2 (8), ErbB2 (9) and Bcl-2 (10) and acetylate or phosphorylate p53 $(3,11)$, inducing cell apoptosis or proliferation inhibition. However, the cell pathways through which aspirin exerts its anticancer effects are not well understood and require further elucidation.

Apoptosis in anticancer strategies is a multi-step process involving the activation of caspases, a family of cysteine proteases. There are two types of apoptotic caspases: initiators (caspases-2, -8, -9 and -10) and effectors (caspases-3,-6 and -7). Initiator caspases cleave inactive pro-forms of effector caspases to activate them and then effector caspases catalyze the specific cleavage of other key cellular proteins to trigger the apoptotic process. In aspirin-induced cancer cell apoptosis, caspase-3 and other caspase family members were previously demonstrated to be crucial upregulated factors $(4,10,12,13)$.

The transcription factor AP-2 family, which consists of AP- $2 \alpha, \beta, \gamma, \delta$ and $\varepsilon$, regulates the transcription of numerous genes involved in mammalian development, the cell cycle, cell proliferation, apoptosis and carcinogenesis by binding to the genes' promoter regions $(14,15)$. Several reports have suggested that AP- $2 \alpha$ and AP- $2 \gamma$ are marked functional activators for ErbB2 overexpression in mammary carcinoma $(16,17)$, while ErbB2 overexpression is associated with increased tumorigenicity, enhanced metastasis, poor prognosis and decreased chemosensitivity (18).

The aim of the present study was to demonstrate whether aspirin induces apoptosis in MDA-MB-453 cells by upregulating caspase-3 expression and activity. Caspase-3 was found to be directly and negatively regulated by AP- $2 \alpha$, which is degraded following aspirin treatment. The caspase- 3 pathway appeared to participate in aspirin-induced apoptosis of MDA-MB-453 cells through the downregulation of AP- $2 \alpha$. 


\section{Materials and methods}

Plasmids, siRNA and reagents. The full-length coding region of AP-2 $\alpha$ was cloned into a pCMV-Myc plasmid (Clontech, Mountain View, CA, USA). The promoter region (nt -799 to $\mathrm{nt}+93$ from the transcription start codon) of caspase- 3 was generated by PCR (19) and cloned into the luciferase reporter plasmid pTAL-luc (Clontech), denoted pTAL-luc-caspase-3. The primer sequences used were: 5'-CGGCTAGCC TTTTTCCTCATGATGTT-3' (forward) and 5'-GAAGATC TGCCTCCTCATACCTTCTAC-3' (reverse). Single or double AP- $2 \alpha$ binding site mutants were created by site-directed mutation and denoted as pTAL-luc-M1, -M2 and -M3. Two specific small interfering RNAs (siRNAs) against AP-2 $\alpha$ were purchased from GenePharma Co. (Shanghai, China). The two siRNA sense sequences are 5'-UUUCUCAACCGACAA CAUUtt-3' and 5'-CGAAGUCUUCUGUUCAGUUtt-3'. Aspirin was purchased from Sigma-Aldrich (St. Louis, MO, USA) and solubilized in water.

Cell culture, transfection and treatment. MDA-MB-453 cells were cultured in DMEM (Gibco-BRL, Carlsbad, CA, USA) supplemented with $10 \%$ fetal bovine serum (FBS; Gibco-BRL), $2 \mathrm{mM}$ L-glutamine, $100 \mathrm{U} / \mathrm{ml}$ penicillin and $100 \mu \mathrm{g} / \mathrm{ml}$ streptomycin at $37^{\circ} \mathrm{C}$ in a $5 \% \mathrm{CO}_{2}$ incubator. Cells were cultured to $80 \%$ confluence and transiently transfected using Lipofectamine 2000 (Invitrogen, Carlsbad, CA, USA) according to the manufacturer's instructions. Aspirin treatment proceeded at $90 \%$ confluence continuously in low-serum ( $0.5 \% \mathrm{FBS})$ medium for $24 \mathrm{~h}$.

Cell proliferation assay. Inhibition of cell proliferation was evaluated by the MTT assay (Sigma-Aldrich) according to the manufacturer's instructions. Briefly, MDA-MB-453 cells were placed in 24-well plates at a density of $1 \times 10^{5}$ cells/well in $450-\mu 1$ medium and $24 \mathrm{~h}$ after attachment, the cells were treated with $0-20 \mathrm{mM}$ aspirin for a further $24 \mathrm{~h}$. Then, $50 \mu \mathrm{l}$ of MTT $(5 \mathrm{mg} / \mathrm{ml}$ in PBS) solution were added to each well and the cells were incubated for a further $4 \mathrm{~h}$, followed by the addition of $150 \mu$ l of dimethyl sulfoxide (DMSO; Sigma-Aldrich)/ well. The cells were left for $30 \mathrm{~min}$ at room temperature to allow color development. Absorbance values were determined using an enzyme-linked immunosorbent assay (ELISA) reader (Model 680; Bio-Rad, Hercules, CA, USA) at $570 \mathrm{~nm}$.

Flow cytometric analysis of apoptosis. Apoptotic and total dead cells were stained by the Annexin V-FITC/propidium iodide (PI) detection kit (Bender MedSystems, Vienna, Austria) according to the manufacturer's instructions. In brief, MDA-MB-453 cells $\left(1 \times 10^{5}\right.$ cells/well) were washed with $1 \mathrm{X}$ Annexin V binding buffer and stained with Annexin V $(5 \mu \mathrm{l})$ and PI $(10 \mu \mathrm{l})$ for $15 \mathrm{~min}$ at room temperature in the dark. Following the addition of $400 \mu \mathrm{l}$ of binding buffer, cells were analyzed by flow cytometry (FACSCalibur; BD Biosciences, San Jose, CA, USA).

Caspase- 3 activity assay. The activity of caspase- 3 was determined using the Caspase-3 Activity Detection kit (Beyotime Institute of Biotechnology, Haimen, China). To evaluate the activity of caspase- 3 , cell lysates were prepared following the designated treatment. Assays were performed in 96-well plates by incubating $10 \mu \mathrm{l}$ protein of cell lysate/sample with $10 \mu \mathrm{l}$ caspase-3 substrate (Ac-DEVD-pNA, $2 \mathrm{mM}$ ) in $80 \mu \mathrm{l}$ of reaction buffer [1\% NP-40, $20 \mathrm{mM}$ Tris- $\mathrm{HCl}(\mathrm{pH} 7.5), 137 \mathrm{mM}$ $\mathrm{NaCl}$ and $10 \%$ glycerol] at $37^{\circ} \mathrm{C}$ for $5 \mathrm{~h}$. Absorbance values were determined using an enzyme-linked immunosorbent assay (ELISA) reader (Model 680; Bio-Rad) at $405 \mathrm{~nm}$.

The detailed procedure including the standard curve preparation was described in the manufacturer's instructions. All the experiments were performed in triplicate.

RNA extraction, semi-quantitative RT-PCR and real-time $P C R$. Total RNA extraction, RT- and real-time PCR were performed as described previously (9). The real-time PCR primers used were: AP2 $\alpha$ 5'-CTCAACCGACAACATTCC-3' (forward) and 5'-CGGTGAACTCTTTGCATATC-3' (reverse) (20); caspase-3 5'-CAGTGGAGGCCGACTTC TTG-3' (forward) and 5'-TGGCACAAAGCGACTGGAT-3' (reverse) (21). For semi-quantitative RT-PCR, DNA was amplified under the following conditions: denaturation at $94^{\circ} \mathrm{C}$ for $30 \mathrm{sec}$, annealing at $55^{\circ} \mathrm{C}$ for $30 \mathrm{sec}$ and extension at $72^{\circ} \mathrm{C}$ for $30 \mathrm{sec}$ and the number of cycles for amplification was 21-25. PCR products were separated by electrophoresis on a $2 \%$ agarose gel and visualized by ethidium bromide staining.

Western blotting. Cells were lysed in RIPA buffer with protease inhibitors (Sigma-Aldrich). Equal amounts of protein were separated on a $10 \%$ SDS-polyacrylamide gel and transferred onto a PVDF membrane (Millipore, Billerica, MA, USA). After blocking, the PVDF membranes were washed three times for $10 \mathrm{~min}$ with TBST at room temperature and incubated for 1-2 $\mathrm{h}$ at room temperature with TBST-diluted primary antibodies, anti-AP2 $\alpha(1: 500)$ and anti-caspase- 3 (1:500; Santa Cruz Biotechnology, Inc., Santa Cruz, CA, USA). Following extensive washing, the membranes were further incubated with the secondary peroxidase-labeled antibodies $(1: 2,000)$ in $5 \%$ non-fat dry milk/TBST for $1 \mathrm{~h}$. The membranes were again washed three times for $10 \mathrm{~min}$ with TBST at room temperature, immunoreactivity was visualized using the enhanced chemiluminescence ECL kit (Pierce Biotechnology, Rockford, IL, USA) and the membranes were exposed to Kodak film. The membranes were then stripped and reprobed with anti-GAPDH antibody (1:1,000; Santa Cruz Biotechnology, Inc.) as a loading control.

Chromatin immunoprecipitation (ChIP) assay. The ChIP assay was performed using the EZ ChIP ${ }^{\mathrm{TM}}$ kit (Millipore) according to the manufacturer's instructions, as previously described (22). The antibodies used included anti-AP2 $\alpha$ antibody (Santa Cruz Biotechnology, Inc.) and normal rabbit IgG (Millipore). The caspase-3 promoter ChIP primers used in the present study were: 5'-AACACAGCATGCGTGGACCT-3' (forward) and 5'-GCCTCCTCATACCTTCTAC-3' (reverse).

Luciferase reporter assays. MDA-MB-453 cells were seeded at $5 \times 10^{5}$ cells/well in 12 -well plates and transfected at $80 \%$ confluence using Lipofectamine 2000 reagent according to the manufacturer's instructions. After $24 \mathrm{~h}$ of transfection, the luciferase activity was measured using the luciferase reporter assay system (Promega, Madison, WI, USA). 
A

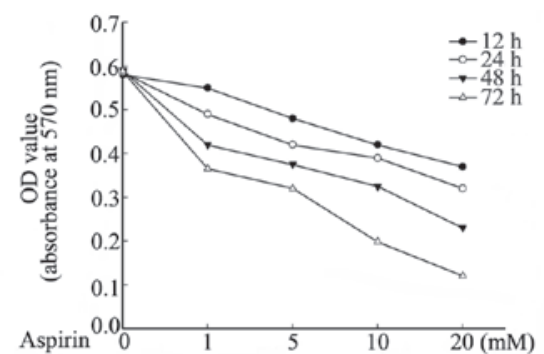

B
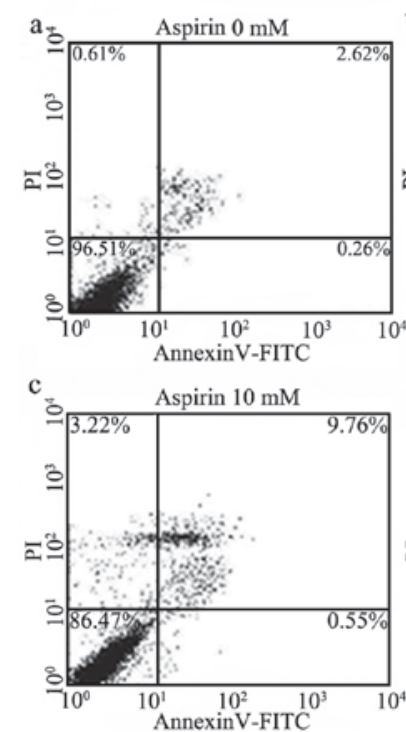
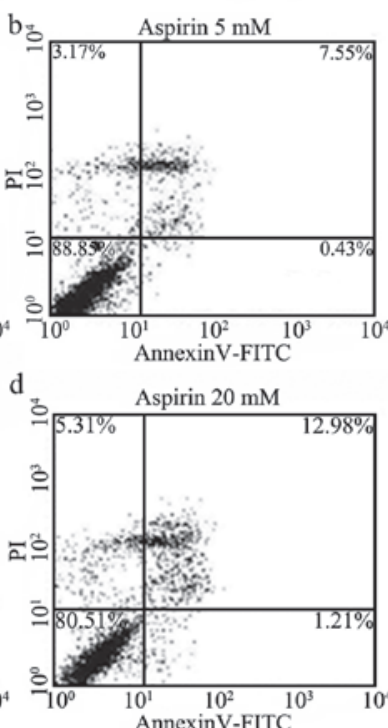

C

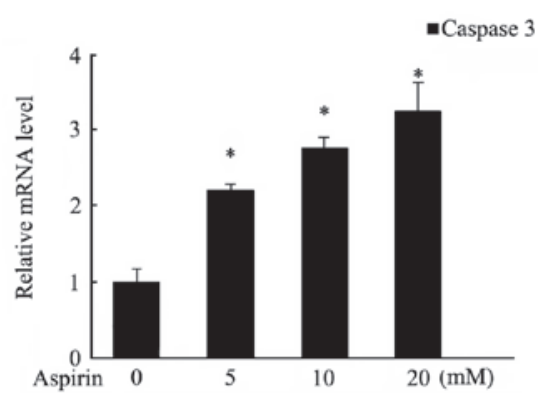

D

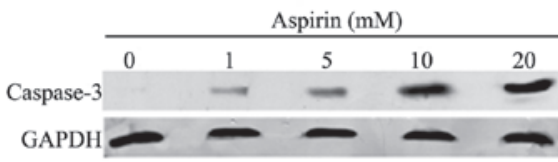

$\mathbf{E}$

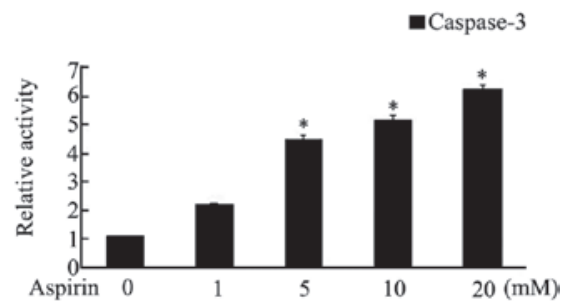

Figure 1. Aspirin induces MDA-MB-453 cell apoptosis by upregulating the expression and activity of caspase-3. (A) MTT assays in MDA-MB-453 cells treated with aspirin at various time points. (B) Annexin V-FITC/PI assays in MDA-MB-453 cells treated with aspirin at 0,5, 10 and 20 mM. Numbers in the respective quadrant profiles indicate the percentage of cells present in this area. (C) Quantitative real-time PCR of the mRNA level of caspase-3 in MDA-MB-453 cells treated with aspirin as indicated. (D) Western blot analysis of the protein expression of caspase-3 in MDA-MB-453 cells treated with aspirin as indicated with GAPDH as a loading control. (E) Colorimetric assay of the activity of caspase-3 in indicated cells treated with aspirin, where each value is expressed as the ratio of caspase- 3 activation level to the control level $(0 \mathrm{mM}) .{ }^{*} \mathrm{P}<0.05$ as compared with the control.

Statistical analysis. Results of bar graphs were expressed as the mean \pm SD obtained from three independent experiments. Statistical differences were evaluated using the Student's t-test. $\mathrm{P}<0.05$ was considered to indicate statistically significant differences.

\section{Results}

Aspirin induced apoptosis and upregulated the expression and activity of caspase-3 in MDA-MB-453 cells. To evaluate the effect of aspirin on the proliferation of human MDA-MB-453 breast cancer cells, the MTT assay was used and the results indicated that aspirin decreased cell proliferation in a dose- and time-dependent manner (Fig. 1A). A significant antiproliferative effect of aspirin appeared following treatment with $20 \mathrm{mM}$ for various time periods. The percentage of viable cells decreased to $62.38 \%$ under treatment with $20 \mathrm{mM}$ of aspirin for $24 \mathrm{~h}$ and then to $18.97 \%$ after $72 \mathrm{~h}$ treatment, compared with the controls. To investigate whether aspirin induced cell apoptosis and death, cells were treated with various concentrations $(0-20 \mathrm{mM})$ of aspirin for $24 \mathrm{~h}$ and then subjected to Annexin V/PI-based flow cytometry. Exposure to aspirin significantly affected apoptosis in the MDA-MB-453 cells (Fig. 1B). When exposed to $20 \mathrm{mM}$ aspirin for $24 \mathrm{~h}$, the percentage of apoptotic cells reached $12.98 \%$, notably higher than the untreated cells. As other studies have described upregulation of the expression and activity of caspase- 3 in gastric, cervical and prostate cancer cell apoptosis $(12,13,23,24)$, the expression of caspase- 3 was investigated by real-time PCR and western blotting. As shown in Fig. 1C and D, the mRNA and protein levels of caspase-3 were increased in a dose-dependent manner when exposed to $0-20 \mathrm{mM}$ aspirin for $24 \mathrm{~h}$. Moreover, to investigate the expression of activated caspase-3, the activity of caspase- 3 was evaluated and the results showed that activity was increased in a similar dose-dependent manner in response to aspirin treatment for $24 \mathrm{~h}$ (Fig. 1E). These data suggested that aspirin induced apoptosis in MDA-MB-453 cells via caspase-3 upregulation.

$A P 2 \alpha$ directly binds to the promoter region of caspase- 3 in $M D A-M B-453$ cells. Since the transcription level of caspase-3 is upregulated in aspirin-treated MDA-MB-453 cells, the promoter region of caspase- 3 was analyzed by the JASPAR and MatInspector programs. As shown in Fig. 2A, two AP-2 $\alpha$ consensus DNA-binding sites in the promoter region of caspase- 3 (nt -901 to $n t+200$ from transcription start codon) were observed, nt -227 and $n t-107$. As AP- $2 \alpha$ is important in mammary carcinoma and highly expressed in MDA-MB-453 cells $(16,17,25)$, the direct binding of AP- $2 \alpha$ to the caspase-3 promoter was demonstrated by ChIP experiments 
A

-901TTAATTGACGCCGTCTTTGCAGAAGGACCTCTCGGCCCAAACCAGGCTTC -851TTTTCCTCTTCTTCACTCCCTCTGGATTGGTTCGTTAATCTCTTTGCCCC -801ATCTCITTTTCCTCATGATG TTAAATGCTACTTTGTTGTGGGATGCTTAA -751CTTGTAACATTTATATGTGGATTAAGTACACTACTATGATGCGTGAGCGG -701CTTGAGCCTGTGTGTCAGGCTCTGACTACCCAGTGACCAGCAAGTGCTGA -651GAACTGCCTCCTTGGGAACTCCACGCAGCTCGTGGCTTTTGTGATCGAAA -601GAGCGTCCACAGCAGCCTGACATGGTGGAAAACACAGCATGCGTGGACCT -551GGTTATTTCTAGCCTGGCACCCTGATGACAGGAGGTTAAGGCTTTTGATT -501TAGGGAGAGAGACCTGAGGGTAACCCAAAGAAAGGCCTCAGGTCATCGCA -451GAGGAGACAGAAATTACCGGTGGGCGTCCCGGCCTGCTCCTCTTCTTCCC -401AGAGCTCAAACTGGGCAGCAGGCCTGGCTTTGTCTCCCTTCTAGGTACCC -351GTTCGCCCTGCCGCGGTTTCCAAGTCTCCCTCAATTTCCCTCCAGAATCC 作作 -251TCCGGGGCGGGGGAGCCGGGCCGA GCCTCAGGGCGGGACCAAAGCGGGAG -201GACTGGCGCGGGACGCGAGGGCGGGACCAGGGCGTGAGGACGCGGGGCGC -151GGGGGCGGGGCGCGCTGCGGTGGGCGGGCGCCTCCTGGGGCCCA GCCTGC -101 बुGOTGGGTTCTCGGGCGGGCGGGAGCTAGGGGACGGGCCGCAGCGGAGGC -51 GGCGCGAGGGGCCGACAGGGGCGGGCGGGAGGCGGGGCTGGCGCTGGAGC

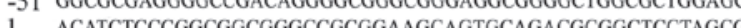
1 ACAT 51 GATGGGTGCTATTGTGAGGCGGTTGTAGAAGGTATGAGGAGGCTGTGCGG 101 TGACTGCCGCCCTCGCTCCGGGCCGTCAGCCCTGCCCCCGCTCCCTGCGT

151 TCTCCCAGGCCCTGCGCCCGCCCGGGTACCTGCCCGGTCTGGAAGGCGG

B

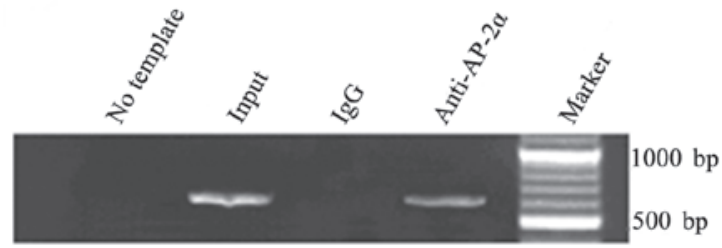

C
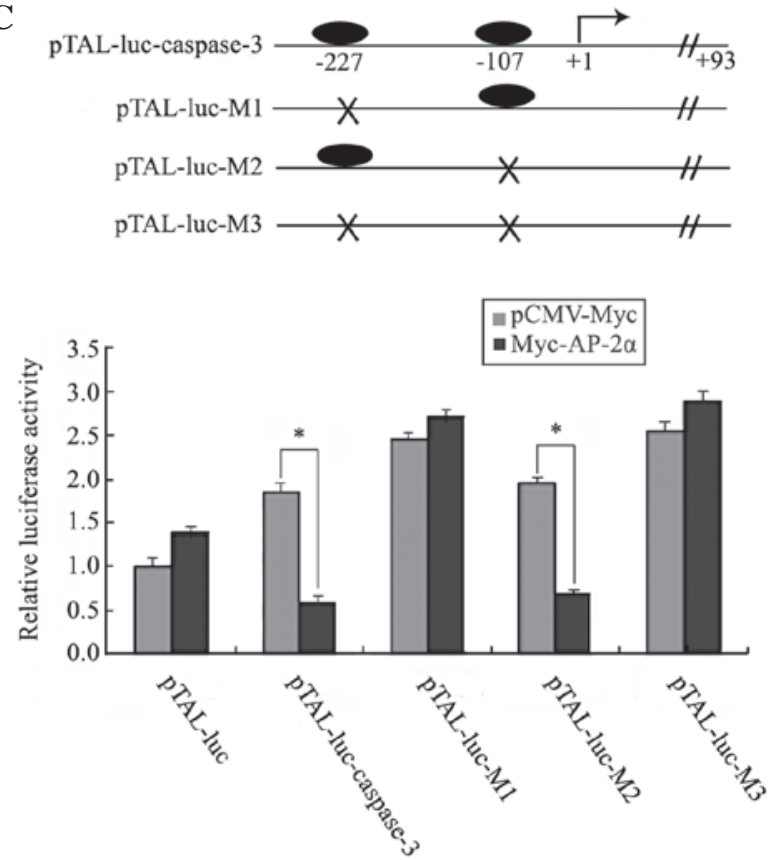

Figure 2. AP2 $\alpha$ directly binds the caspase-3 promoter region. (A) AP2 $\alpha$ binding sites were predicted by the programs JASPAR and MatInspector and are marked with black boxes. (B) ChIP assay for the binding of AP2 $\alpha$ to the caspase-3 promoter. Genomic DNA (input) without immunoprecipitation was used as a positive control. (C) Luciferase reporter assay for determining the precise binding sites of AP2 $\alpha$ on the caspase-3 promoter; the wild-type binding sites of AP2 $\alpha$ are indicated by ovals, and the mutated sites are indicated by crosses $(x)$. The data in the bar charts are the mean $\pm \mathrm{SD}$ of three independent experiments. ${ }^{*} \mathrm{P}<0.05$ as compared with the control.

$\mathbf{A}$

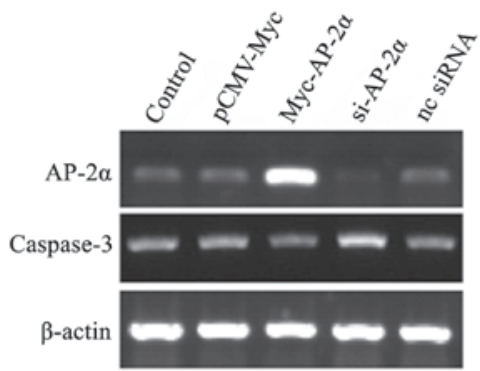

B

Control pCMV-Myc Myc-AP-2 $\alpha$ si-AP-2 $\alpha$ nc siRNA

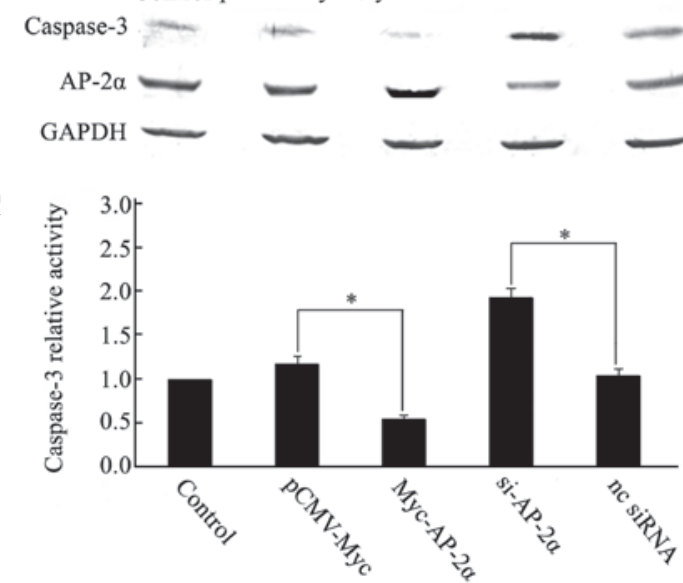

Figure 3. AP $2 \alpha$ negatively regulates caspase- 3 transcription. MDA-MB-453 cells were transfected as follows: control, empty plasmid (pCMV-Myc), AP2 $\alpha$ overexpression plasmid (Myc-AP2 $\alpha$ ), AP $2 \alpha$ siRNA and siRNA negative control (nc). (A) Semi-quantitative PCR of the mRNA level of caspase-3. (B) Western blot analysis of the protein expression of caspase-3 with GAPDH as a loading control. (C) Colorimetric assay of the activity of caspase-3, where each value is expressed as the ratio of the caspase- 3 activation level to the control level. The data in the bar charts represent the mean \pm SD of three independent experiments. ${ }^{*} \mathrm{P}<0.05$ compared with the control.
(Fig. 2B). The predicted band was detected in the input and AP- $2 \alpha$-ChIP-derived DNA samples, but not in the control IgG-ChIP-derived DNA samples. To confirm the precise AP- $2 \alpha$ binding sites in the caspase- 3 promoter, the promoter region (nt -799 to nt +93) of caspase-3 was used for a luciferase reporter assay. Wild-type and mutant promoter luciferase plasmids, including pTAL-luc-caspase-3, pTAL-luc-M1 (nt -227), -M2 (nt -107) and -M3 (nt -227 and nt -107), were constructed and co-transfected with pCMV-Myc or pCMV-Myc-AP-2 $\alpha$ plasmids, independently. As shown in Fig. 2C, compared with the control, overexpression of AP- $2 \alpha$ depressed the transcriptional activities of the constructs pTAL-luc-caspase-3 and pTAL-luc-M2 by $\sim 3$-fold. However, pTAL-luc-M1 and -M3 eliminated the effect of AP- $2 \alpha$, suggesting that AP- $2 \alpha$ directly bound to the caspase- 3 promoter at the nt -227 site.

AP2 $\alpha$ negativelyregulated caspase-3 in MDA-MB-453 cells. To determine whether this binding conferred positive or negative regulation on caspase- 3 , the expression of AP- $2 \alpha$ was knocked down in MDA-MB-453 cells by siRNA and the expression of caspase-3 was detected using semi-quantitative RT-PCR and western blotting. Cells transfected with siRNAs against AP- $2 \alpha$ showed clear increases in caspase-3 mRNA (Fig. 3A) and protein levels (Fig. 3B). Expression of the activated caspase-3 was also enhanced, as determined by a caspase- 3 activity assay (Fig. 3C). However, overexpression of AP-2 $\alpha$ decreased the expression levels (Fig. 3A and B) and activity (Fig. 3C) of caspase-3, whereas the controls did not change.

Aspirin induced the degradation of AP-2 $\alpha$ through the proteasome pathway in MDA-MB-453 cells. To investigate whether AP- $2 \alpha$ responds to aspirin treatment in MDA-MB-453 cells, 
A

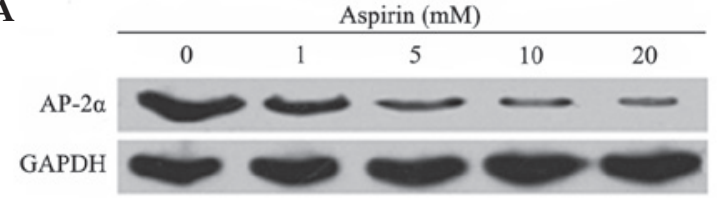

C

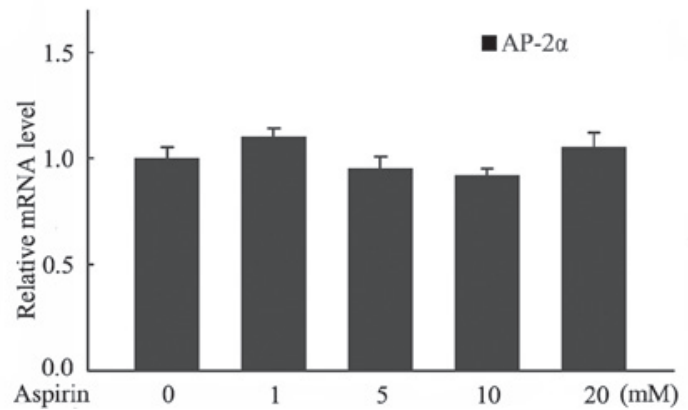

$\mathbf{B}$

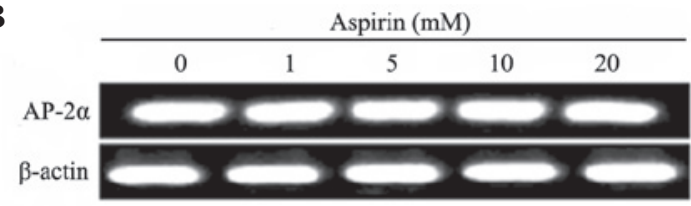

D

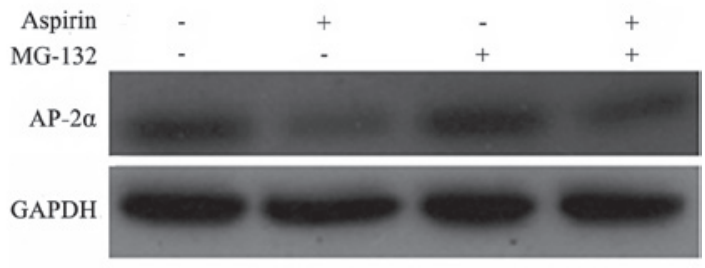

Figure 4. Aspirin induces AP2 $\alpha$ degradation. MDA-MB-453 cells were incubated with 0-20 mM aspirin for 24 h. (A) Western blot analysis of AP2 $\alpha$ protein expression with GAPDH as a loading control. (B) Semi-quantitative PCR of the mRNA level of AP2 $\alpha$ with $\beta$-actin as a loading control. (C) Real-time PCR of the mRNA level of AP2 $\alpha$. (D) Western blot analysis of the aspirin-induced proteasome-mediated degradation of AP2 $\alpha$. Cells were incubated with the MG132 proteasome inhibitor $(20 \mu \mathrm{M})$ for $5 \mathrm{~h}$ prior to aspirin $(10 \mathrm{mM})$ treatment for $24 \mathrm{~h}$. The data in the bar charts are the mean $\pm \mathrm{SD}$ of three independent experiments.

the AP-2 $\alpha$ expression levels of mRNA and protein were evaluated. The results showed that the protein level of AP- $2 \alpha$ was downregulated in a dose-dependent manner in MDA-MB-453 cells following exposure to various concentrations of aspirin for 24 h (Fig. 4A). However, no significant changes were observed in the mRNA levels in the semi-quantitative and real-time PCR experiments (Fig. 4B and C). It appeared that aspirin did not affect AP- $2 \alpha$ expression at the transcriptional level.

A previous study reported that aspirin treatment affects protein proteasomal degradation in breast cancer cells (26). MG132, a proteasome inhibitor, was used to evaluate whether it was able to block the degradation of AP- $2 \alpha$ in MDA-MB-453 cells following aspirin treatment. Cells were pre-incubated with MG132 $(20 \mu \mathrm{M})$ for $5 \mathrm{~h}$ prior to aspirin $(10 \mathrm{mM})$ treatment for $24 \mathrm{~h}$ and cell extracts were used for western blotting to detect AP- $2 \alpha$ expression. As shown in Fig. 4D, the degradation of AP- $2 \alpha$ protein was blocked in the presence of MG132, suggesting that aspirin induced AP- $2 \alpha$ degradation via a proteasome-mediated pathway.

\section{Discussion}

Aspirin has multiple effects and is involved in various cellular processes. In the last few years, several studies have shown that aspirin inhibits cancer cell proliferation and induces apoptosis $(3,11,24)$, which is consistent with the present results that aspirin exhibits the same activity in MDA-MB-453 breast cancer cells in a dose- and time-dependent manner. Although the inhibition of certain proteins, such as $\mathrm{COX}$ and $\mathrm{NF}-\kappa \mathrm{B}$, may contribute to the anticancer effects of aspirin $(8,27)$, at present the pathways leading to these effects remain to be determined.

In the present study, several novel observations were reported, including a mechanism by which aspirin may exert its anticancer effects. The present results showed that the expression and activity of caspase- 3 were upregulated following aspirin treatment, suggesting that the induction of apoptosis by aspirin in MDA-MB-453 cells may depend on the caspase pathway and caspase- 3 was regulated at its transcriptional level. Promoter analysis then revealed that AP- $2 \alpha$ may be a regulator of caspase-3. Considering the important roles of AP-2 $\alpha$ in breast cancer and its high expression levels in several breast cancer cell types including MDA-MB-453 (16,25), further experiments were performed to study the association between AP- $2 \alpha$ and caspase-3. The data showed that AP- $2 \alpha$ negatively regulates caspase- 3 transcription via binding to the promoter region of caspase-3, thus downregulating the expression of caspase-3 and decreasing its activity. The expression of AP-2 $\alpha$ in cells treated with aspirin was then evaluated. The present results have shown that AP- $2 \alpha$ was downregulated following aspirin treatment at the protein but not mRNA level. Aspirin has been reported to affect protein degradation through the proteasome pathway (26). To assess whether this downregulation of AP-2 $\alpha$ depends on the proteasome pathway, MG132 was selected to block the proteasome pathway. MG132 abrogated the aspirin-induced reduction of AP- $2 \alpha$.

From the present study, a working model may be proposed. In this model, AP- $2 \alpha$ negatively regulates caspase- 3 by binding to its proximal promoter region in AP- $2 \alpha$-positive cancer cells. Aspirin promotes the proteasome pathway-dependent degradation of AP-2 $\alpha$, which increases the expression of apoptotic effector caspase-3, leading to an increase in activated caspase- 3 and finally causing cell apoptosis. This suggests a novel mechanism for aspirin in breast cancer treatment.

AP- $2 \alpha$ acts as an oncogene, which is consistent with previous reports $(16,17,25,28)$. However, there are also several lines of evidence indicating that AP- $2 \alpha$ may act as a tumor suppressor gene. Overexpression of AP-2 $\alpha$ induces apoptosis in a number of cancer cells (29-31). AP-2 $\alpha$ has been shown to act as both a tumor suppressor and an oncogene in various cancer types that may depend on the expression level and signaling pathways AP- $2 \alpha$ is involved in. The controversy concerning the role of AP- $2 \alpha$ reflects the complexity of the cell signaling pathways. However, a number of critical questions remain to be answered in order to understand the mechanisms of aspirin-induced apoptosis. 
In summary, the present study demonstrated for the first time that aspirin upregulates caspase-3 activity through the downregulation of AP- $2 \alpha$ gene expression, leading to the apoptosis of human breast cancer cells. The present findings also suggest that aspirin is a potentially powerful therapeutic agent for various AP- $2 \alpha$-dependent human cancers.

\section{Acknowledgements}

The present study was supported in part by the National Natural Science Foundation of China (Nos. 30972624 and 81172112), New Century Excellent Talents in University (NCET-10-0145), Science and Technology Department of Hunan Province (2011FJ3140, 2011TT2008), Scientific Research Fund of Hunan Provincial Education Department (11A072) and China Postdoctoral Science Foundation (2012M511380).

\section{References}

1. Qiao L, Hanif R, Sphicas E, Shiff SJ and Rigas B: Effect of aspirin on induction of apoptosis in HT-29 human colon adenocarcinoma cells. Biochem Pharmacol 55: 53-64, 1998.

2. Kutuk $\mathrm{O}$ and Basaga $\mathrm{H}$ : Aspirin inhibits TNFalpha- and IL-1-induced NF-kappaB activation and sensitizes HeLa cells to apoptosis. Cytokine 25: 229-237, 2004.

3. Alfonso LF, Srivenugopal KS, Arumugam TV, Abbruscato TJ, Weidanz JA and Bhat GJ: Aspirin inhibits camptothecin-induced p21 $1^{\mathrm{CIP1}}$ levels and potentiates apoptosis in human breast cancer cells. Int J Oncol 34: 597-608, 2009.

4. Chattopadhyay M, Goswami S, Rodes DB, et al: NO-releasing NSAIDs suppress NF- $\kappa$ B signaling in vitro and in vivo through S-nitrosylation. Cancer Lett 298: 204-211, 2010.

5. Zhang Z, Huang L, Zhao W and Rigas B: Annexin 1 induced by anti-inflammatory drugs binds to NF-kappaB and inhibits its activation: anticancer effects in vitro and in vivo. Cancer Res 70 2379-2388, 2010.

6. Zhou H, Huang L, Sun Y and Rigas B: Nitric oxide-donating aspirin inhibits the growth of pancreatic cancer cells through redox-dependent signaling. Cancer Lett 273: 292-299, 2009.

7. Rigas B and Williams JL: NO-donating NSAIDs and cancer: an overview with a note on whether NO is required for their action. Nitric Oxide 19: 199-204, 2008.

8. Harris RE, Beebe-Donk J, Doss H and Burr Doss D: Aspirin, ibuprofen, and other non-steroidal anti-inflammatory drugs in cancer prevention: a critical review of non-selective COX-2 blockade (Review). Oncol Rep 13: 559-583, 2005.

9. Xiang S, Sun Z, He Q, Yan F, Wang Y and Zhang J: Aspirin inhibits ErbB2 to induce apoptosis in cervical cancer cells. Med Oncol 27: 379-387, 2010.

10. Kim KM, Song JJ, An JY, Kwon YT and Lee YJ: Pretreatment of acetylsalicylic acid promotes tumor necrosis factor-related apoptosis-inducing ligand-induced apoptosis by down-regulating BCL-2 gene expression. J Biol Chem 280: 41047-41056, 2005.

11. Luciani MG, Campregher $\mathrm{C}$ and Gasche $\mathrm{C}$ : Aspirin blocks proliferation in colon cells by inducing a G1 arrest and apoptosis through activation of the checkpoint kinase ATM. Carcinogenesis 28: 2207-2217, 2007.

12. Royle JS, Ross JA, Ansell I, Bollina P, Tulloch DN and Habib FK: Nitric oxide donating nonsteroidal anti-inflammatory drugs induce apoptosis in human prostate cancer cell systems and human prostatic stroma via caspase-3. J Urol 172: 338-344, 2004.
13. Gu Q, Wang JD, Xia HH, et al: Activation of the caspase-8/Bid and Bax pathways in aspirin-induced apoptosis in gastric cancer. Carcinogenesis 26: 541-546, 2005.

14. Hilger-Eversheim K, Moser M, Schorle H and Buettner R: Regulatory roles of AP-2 transcription factors in vertebrate development, apoptosis and cell-cycle control. Gene 260: 1-12, 2000.

15. Turner BC, Zhang J, Gumbs AA, et al: Expression of AP-2 transcription factors in human breast cancer correlates with the regulation of multiple growth factor signalling pathways. Cancer Res 58: 5466-5472, 1998.

16. Bosher JM, Williams T and Hurst HC: The developmentally regulated transcription factor AP-2 is involved in c-erbB-2 overexpression in human mammary carcinoma. Proc Natl Acad Sci USA 92: 744-747, 1995.

17. Bosher JM, Totty NF, Hsuan JJ, Williams T and Hurst HC: A family of AP-2 proteins regulates c-erbB-2 expression in mammary carcinoma. Oncogene 13: 1701-1707, 1996.

18. Agus DB, Akita RW, Fox WD, et al: Targeting ligand-activated ErbB2 signaling inhibits breast and prostate tumor growth. Cancer Cell 2: 127-137, 2002.

19. Sudhakar C, Jain N and Swarup G: Sp1-like sequences mediate human caspase-3 promoter activation by p73 and cisplatin. FEBS J 275: 2200-2213, 2008.

20. Hubert MA, Sherritt SL, Bachurski CJ and Handwerger S: Involvement of transcription factor NR2F2 in human trophoblast differentiation. PLoS One 5: e9417, 2010.

21. Ji BC, Hsu WH, Yang JS, et al: Gallic acid induces apoptosis via caspase- 3 and mitochondrion-dependent pathways in vitro and suppresses lung xenograft tumor growth in vivo. J Agric Food Chem 57: 7596-7604, 2009.

22. Ailan H, Xiangwen X, Daolong R, et al: Identification of target genes of transcription factor activator protein 2 gamma in breast cancer cells. BMC Cancer 9: 279, 2009.

23. Power JJ, Dennis MS, Redlak MJ and Miller TA: Aspirin-induced mucosal cell death in human gastric cells: evidence supporting an apoptotic mechanism. Dig Dis Sci 49: 1518-1525, 2004.

24. Lee SK, Park MS and Nam MJ: Aspirin has antitumor effects via expression of calpain gene in cervical cancer cells. J Oncol 2008: 285374, 2008.

25. Begon DY, Delacroix L, Vernimmen D, Jackers P and Winkler R: Yin Yang 1 cooperates with activator protein 2 to stimulate ERBB2 gene expression in mammary cancer cells. J Biol Chem 280: 24428-24434, 2005.

26. Lu M, Strohecker A, Chen F, et al: Aspirin sensitizes cancer cells to TRAIL-induced apoptosis by reducing survivin levels. Clin Cancer Res 14: 3168-3176, 2008.

27. McCarty MF and Block KI: Preadministration of high-dose salicylates, suppressors of NF-kappaB activation, may increase the chemosensitivity of many cancers: an example of proapoptotic signal modulation therapy. Integr Cancer Ther 5: 252-268, 2006.

28. Zhu CH and Domann FE: Dominant negative interference of transcription factor AP-2 causes inhibition of ErbB-3 expression and suppresses malignant cell growth. Breast Cancer Res Treat 71: 47-57, 2002.

29. Wajapeyee N and Somasundaram K: Cell cycle arrest and apoptosis induction by activator protein 2alpha (AP-2alpha) and the role of p53 and p21WAF1/CIP1 in AP-2alpha-mediated growth inhibition. J Biol Chem 278: 52093-52101, 2003.

30. Müller FU, Loser K, Kleideiter U, et al: Transcription factor AP-2alpha triggers apoptosis in cardiac myocytes. Cell Death Differ 11: 485-493, 2004.

31. Wajapeyee N, Britto R, Ravishankar HM and Somasundaram K: Apoptosis induction by activator protein 2alpha involves transcriptional repression of Bcl-2. J Biol Chem 281: 16207-16219, 2006. 